\title{
Bi-parametric MRI/TRUS fusion targeted repeat biopsy after systematic 10-12 core TRUS-guided biopsy reveals more significant prostate cancer especially in anteriorly located tumours
}

\section{Michael Häggman}

Uppsala Universitet Medicinska fakulteten

\section{Mats Ahlberg}

Uppsala Universitet Medicinska fakulteten

\section{Pär Dahlman}

Uppsala Universitet Medicinska fakulteten

\section{Per Liss}

Uppsala Universitet Medicinska fakulteten

\section{Rafaele Cantera Ahlman}

Uppsala Universitet Medicinska fakulteten

\section{Pontus Röbeck}

Umea Universitet Medicinska fakulteten

\section{Anca Dragomir}

Akademiska Sjukhuset Klinisk patologi

\section{Håkan Jorulf}

Uppsala Universitet Medicinska fakulteten

\section{Sam Ladjevardi ( $\square$ Sam.ladjevardi@surgsci.uu.se )}

Uppsala Universitet Medicinska och farmaceutiska vetenskapsomradet https://orcid.org/0000-00024000-4159

\section{Research article}

Keywords: Prostate cancer, MRI, Bi-parametric MRI, Fusion guided biopsy, Transrectal ultrasound, Diagnosis

Posted Date: July 6th, 2020

DOI: https://doi.org/10.21203/rs.3.rs-39813/v1

License: (c) (1) This work is licensed under a Creative Commons Attribution 4.0 International License. Read Full License 
Page 2/14 


\section{Abstract \\ Background}

To demonstrate possible advantages with Bi-parametric MRI fusion-guided repeat biopsy over systematic 10-12-core biopsy for diagnosis of prostate cancer.

\section{Methods}

All patients who underwent fusion guided biopsy between February 2015 to February 2017 were included. Four hundred twenty-three consecutive men, with previous systematic10-12-core TRUS-guided biopsy, with suspicion of or low-risk prostate cancer underwent fusion-guided prostate biopsy. The material was retrospectively assessed. In 220 cases no previous cancer was diagnosed and in 203 cases confirmatory fusion guided biopsy was performed prior to active monitoring. MRI was done and classified according to PI-RADS. Region of Interest (ROI) was marked. Systematic biopsy was compared to fusion guided biopsy for detection of cancer, as well as PI-RADS was compared to Gleason score.

\section{Results}

Fusion guided biopsy detected significantly more cancers than systematic $(p<0.001)$. Gleason scores were higher in the fusion biopsy group $(p<0,001)$. PI-RADS score correlated with the presence of cancer in fusion biopsies. PI-RADS 2 yielded 19\% cancer and PI-RADS $356 \%$, PI-RADS 4-5 were cancerous in $83 \%$. Only 3 out of 53 biopsies from PI-RADS 2 lesions showed high grade cancer (Gleason score $4+3$ and higher) compared to 10/133 (7,5\%) from PI-RADS 3 lesions and 54/239 (23\%) from PI-RADS 4-5.

\section{Conclusion}

These results show superior detection rate and grading of bi-parametric MRI/TRUS fusion targeted repeat biopsy over systematic 10-12 core biopsies. Fusion guided biopsy detects more significant cancers despite using fewer cores. There is significant impact on known cases of low-risk PCa with change of risk group for many patients initially selected for active surveillance.

\section{Introduction}

Despite that prostate cancer ( $\mathrm{PCa}$ ) remains one of the most diagnosed cancers among men worldwide ${ }^{1}$ the diagnostic accuracy is still a challenge for both physician and the patient. An accurate verification of significant and potentially lethal PCa, at the same time excluding insignificant PCa poses a clinical problem. 
It is well known that PSA testing and subsequent 10-12 core systematic biopsy leads to both overdiagnosis of clinical insignificant cancer and a risk to miss significant cancer ${ }^{2}$. The systematic biopsies generally sample the dorsal part of the prostate. Even if $60-70 \%$ of tumours are in the peripheral zone ${ }^{3-5}$, a substantial number will be at risk to be missed. Significant number of cases with negative systematic biopsies are later shown to harbour PCa and many PCa diagnosed by systematic biopsy are inaccurately graded leading to repeat biopsies. Each biopsy occasion may also lead to complications. In Sweden $6 \%$ of patients are treated with antibiotics for urinary tract infections after prostate biopsies and cases with resistant bacteria become increasingly common ${ }^{6}$.

MRI plays a crucial role to identify men with a high likelihood of clinically significant PCa who require immediate biopsy. Multiparametric MRI (mpMRI) has been shown to be a valuable tool to achieve a more accurate biopsy sampling ${ }^{7}$. It has also been shown that mpMRI may avoid diagnosing insignificant cancer, which prevents unnecessary biopsies, possibly minimizing the side effects $8-10$. Since $20 \%$ of the tumours are in anterior part of prostate ${ }^{11}$, targeted biopsies can diagnose those lesions in the prostate that are not diagnosed with systematic biopsies ${ }^{12}$. However, it has been shown that targeted biopsies can also miss significant cancer in some cases and the sensitivity of this method needs improvement before it can replace systematic biopsies ${ }^{13}$. A recent randomized study has shown a clear superiority of fusion-guided mpMRI/TRUS biopsies over standard TRUS-guided biopsy ${ }^{14}$.

The added value of DCE MRI in combination with T2-weighted imaging and DWI is controversial ${ }^{15}$. The role of DCE MRI was recently downgraded by the American College of Radiology (ACR) and the European Society of Urogenital Radiology (ESUR) in the updated Prostate Imaging Reporting and Data System (PIRADS) version 2.1. In detail, DWI for the peripheral zone (PZ) and T2-WI for the transition zone (TZ) were respectively considered the dominant sequences to detect clinically significant tumours. In PI-RADS v2, the role of DCE is minor limited to potentially upgrading a PI-RADS 3 lesion in the PZ to PI-RADS $4{ }^{16}$.

It has been shown that the diagnostic accuracy of a bi-parametric MRI (bpMRI) imaging protocol consisting of T2-weighted imaging and DWI is comparable with that of a standard multi-parametric imaging protocol for the detection of clinically significant PCa ${ }^{17}$.

Since, software for fusion by computer processing was developed, in form of rigid and subsequently elastic fusion. Rigid image registration overlays the MRI images onto the TRUS images during the biopsy procedure without adjustment for possible deformation of the prostate due to patient movement or the introduction of the TRUS probe ${ }^{18}$. Elastic registration, however, aims to compensate for this deformation and it is therefore expected to be more accurate than rigid image registration ${ }^{19-21}$. Nevertheless, in studies of men on active surveillance or those with a prior negative biopsy, the cancer detection rate approaches $55 \%$ and up to $94 \%$ in patients with highly suspicious lesions on MRI ${ }^{22}$.

The aims of this study were to evaluate our early clinical experience with bpMRI/TRUS fusion guided biopsy and secondly to evaluate if repeat MRI/TRUS fusion guided biopsy yields more significant PCa as 
compared to initial standard TRUS-guided biopsy as well as improving diagnosis tumours in various locations.

\section{Material And Methods}

\section{Study design and participants}

Inclusion criteria for this retrospective study were men with suspicion of PCa (elevated PSA or palpable tumor at rectal examination), or patients who already were diagnosed with $\mathrm{PCa}$, but with at least one set of systematic biopsies, being considered for active monitoring or patients with strong suspicion of more malignant tumor due to discordance between the PSA level and Gleason score (GS) in systematic biopsies.

Between February 2015 to February 2017, 423 consecutive men, aged 39-78, median 66 with mean PSA 14,3 (SD 1,9-63) who had been evaluated for elevated serum PSA and/or other clinical findings arousing suspicion of PCa were included. All had been subjected to at least one set systematic biopsies, underwent repeat fusion-guided biopsy. Data about age, MRI re-evaluation according to PI-RADS v. 2.0, PSA-value, clinical T-stage, use of 5-alfa-reductase-inhibitor, number of fusion-biopsies and histopathological results were collected.

All together 220 men had no known PCa prior to fusion biopsy, all of them had undergone 1-5 sets of prior negative 10-12 core standard biopsies. Two hundred and three men had low-risk PCa (Gleason score 5-6 or ISUP 1) in their prior biopsies and were candidates for active surveillance of their PCa.

Patients were selected for fusion biopsy according to the following criteria:

- clinical suspicion of PCa (persistent elevated PSA, palpable tumor) but no evidence of PCa after standard TRUS-biopies or

- clinical suspicion of more serious PCa requiring treatment (elevated PSA-level, palpable tumor, highly suspect MRI) but only GS 6 (ISUP 1) or minimal amount of GS 7 (ISUP 2) after standard TRUSbiopsies.

\section{Patients and public involvement:}

The study was designed to improve the diagnosis of prostate cancer in the interest of the patients.

The patients were not specifically involved in the design of the study. They were asked to participate and signed an informed consent. However, our patients were not involved in the conduct, analysis of data or writing the manuscript in other ways.

We will also involve the Swedish Prostate Cancer Patients Union, for possible comments in their newsletter "Prostatanytt. 


\section{MRI assessment}

All patients underwent MRI following the European Society of Urogenital Radiology (ESUR) guidelines; T2 weighted (axial, coronal and sagittal), T1 with large field of view, ADC and high trace value (b1000-2000 $\mathrm{s} / \mathrm{mm} 2$ ). Patients examined in house (55\%) underwent bi-parametric 3T MRI (MRI scanner (Achieva, Philips Healthcare, Best, The Netherlands), high trace value b2000, patients from outside hospitals (45\%) underwent $1,5(75 \%)$ or 3 T MRI (25\%). All patients fulfil examination quality criteria, assigned PI-RADS classification according to PI-RADS v. 2.0. One or more regions of Interest (ROI) were delineated guided by PI-RADS criteria. No patients required repeat imaging. All patients had a complete set of images although artifacts from air in the rectum, hip implants or movement in several cases caused suboptimal image quality most often in diffusion weighted images.

It has been shown in other studies that PI-RADS 2 lesions most likely do not represent clinically significant PCa and therefore, later during the study, we chose PI-RADS 3 as cut-off.

\section{Systematic biopsy}

One to five sets of systematic 10-12 core TRUS guided side fire biopsies were done" free hand" i.e with hand-held TRUS probe., by various physicians at our and at referring centers.

\section{Fusion biopsy}

We performed the fusion biopsy with the Artemis device (Artemis; Eigen, Grass Valley, CA), which allows biopsy site tracking in ultrasound and fusion of real-time ultrasound with MRI, in conjunction with B\&K ultrasonography probe 8818 in end-fire mode. With the Artemis device the position of the US probe is tracked by angle-sensing devices (encoders) built into each joint of the arm. This allows for the reconstruction of the biplanar US into a 3D model, which is then elastically fused with the MRI. Two to seven fusion guided biopsy cores were obtained from 1-3 ROl's in all cases. All fusion biopsies were performed by two experienced urologists. Biopsies were processed as per standard protocol at our Pathology Department and examined by board-certified uropathologists.

One hundred of the 423 patient subsequently underwent robot-assisted radical prostatectomy at our institution and thus had readily available whole-mount sections of the excised prostate for histopathologic evaluation. For this article only, the position of the ROI's vs the location of the biopsied tumor in the specimen was evaluated, other features in the operative specimens will be evaluated and reported in a later publication.

\section{Statistical methods}

The accuracy of TRUS-guided biopsy versus bpMRI/TRUS fusion targeted biopsy in diagnosing PCa (GS $\geq 6$ ) was evaluated. 
MRI images were stratified into groups according to PI-RADS in "2-3" and "4-5". For statistical analysis, Chi-squared 2 test with $95 \%$ confidence interval was used.

Cross tables were made comparing the number of individuals with no PCa and different GS detected by standard biopsy and fusion biopsy. Sign test's (exact binomial tests) was used to test if the number of detected PCa differ between standard biopsy and fusion biopsy and if the number of individuals with GS $\geq 7$ differ between standard biopsy and fusion biopsy.

A comparison of severity for individuals with detected PCa at standard biopsy was done. Here the number of individuals with higher GS in the standard biopsy was compared with the number of individuals with higher GS fusion biopsy. This has also been tested using a sign test.

Cross tables comparing PI-RADS score with GS from standard biopsy and GS from fusion biopsy have been created. The Spearman rank correlation between PI-RADS and GS for standard biopsy and for fusion biopsy has been calculated.

\section{Results}

Out of 220 patients with previous negative on systematic biopsy, fusion biopsy diagnosed PCa in 124 . Among the 203 patients with previous low risk PCa after systematic biopsy, fusion biopsy detected cancer in 158 patients ("Table 1 and 2"). The median number of ROI were 2 (1-3) and the median number of Fusion guided biopsy were 5 (3-7 biopsies). Out of 423 patients, clinically significant (GS $\geq 7$ ) PCa was detected in 190 (45\%) patients.

Among 220 patients with previous negative findings at systematic biopsy, fusion biopsy detected intermediary risk (ISUP 2-3) PCa in 56 patients and high risk (ISUP 4-5) PCa in 34 patients. Among those 203 patients who were initially diagnosed with low risk cancer after systematic biopsy, 62 patients were re-classified after fusion biopsy as intermediary risk (ISUP 2-3) and 8 patients as high-risk cancer (ISUP 45). Among 27 patients who were initially diagnosed with intermediary risk cancer (ISUP 2-3) after standard biopsy, 8 patients were re-classified after fusion biopsy as high risk (ISUP 4-5) (“Table 3"). Upgrading in GS after fusion biopsy occurred in 202 cases, 158 were in concordance and in 63 cases after fusion biopsy the GS were downgraded ( $p$ 冈0.001), (“Table 4").

In total, 51 patients were classified as PI-RADS 2. Seventeen of them had a previous PCa-diagnosis after standard biopsies, 11 patients (22\%) with GS 6 (ISUP 1), and 6 patients (12\%) with GS 7-8 (ISUP 2-4). Fusion biopsies revealed PCa in 10 patients, of which 5 had GS 6 (ISUP1), 3 had GS 7 (ISUP2-3) and one had GS 8 (ISUP4).

One hundred thirty-three patients were classified as PI-RADS 3. In 59 (44\%) of them PCa was diagnosed by standard biopsy, with GS 6 (ISUP1) in 46 (35\%), GS 7a (ISUP2) in 12 (9\%) and GS 8 (ISUP4) in 1. After fusion biopsies PCa was found in 74 (56\%) patients, of which 31 (42\%) had GS 6 (ISUP1), 33 (45\%) had GS 7a (ISUP2) and 9 (12\%) GS 7b (ISUP3) and one with GS 8 (ISUP 4). 
One hundred seventy patients were classified as PI-RADS 4. Previous standard biopsies had shown PCa, in 93 (55\%) of patients: 62 (36\%) with GS 6 (ISUP1), 26 (15\%) with GS 7a (ISUP2) and 5 (3\%) with GS 7b (ISUP3). After fusion-biopsies PCa was found in 142 (84\%) patients: 47 (28\%) had GS 6 (ISUP1), 56 (33\%) had GS 7a (ISUP2), 18 (11\%) had GS 7b (ISUP3), 10 (6\%) had GS 8 (ISUP4), and one had GS 9 (ISUP5). Two patients had no specified GS because 5ARI-treatment.

Thirty-one patients were classified as PIRADS 5 and 12 (32\%) of them had prior PCa-diagnosis after standard biopsies: 9 (29\%) GS 6 (ISUP1), 2 (6\%) GS 7a (ISUP2) and one with GS 7b (ISUP3). After fusion biopsies PCa was found in 30 patients: 5 (17\%) GS 6 (ISUP1), 13 (43\%) GS 7a (ISUP2) and 4 with GS 7b (ISUP3), 1 (3\%) GS 8 (ISUP4), 7 (23\%) GS 9 (ISUP5) (“Tables 5 and 6”). In one patient no cancer was either detected after fusion biopsies.

Out of 220 cases, 34 (15\%) were upgraded from no cancer to low risk and $90(41 \%)$ to intermediate/ high risk cancer by repeat fusion-guided biopsy. Out of 203 cases, 70 (34\%) went from low risk to intermediate/high risk cancer. Thus, 160 cases had their risk group changed to possibly be subjected to curative treatment.

Among all patients who were included in this study MRI/TRUS fusion guided biopsy revealed 228 (54\%) patients with ventral tumors and $195(46 \%)$ patients with dorsally located tumors. Of 203 patients who were preliminary diagnosed with standard biopsy, MRI revealed $45(20 \%)$ patients with ventral tumors and $30(15,4 \%)$ patients with dorsally located tumors. Of 45 patients with ventral low risk cancer MRI/TRUS guided biopsy revealed significant aggressive cancer in 37 (82\%) of patients. Of 30 patients with dorsally located low risk cancer MRI/TRUS guided biopsy revealed 21 (70\%) patients with significant aggressive cancer. In 35 patients were systematic biopsies diagnosed GS $3+3$ (ISUP 1) in insignificant amounts (less than $3 \mathrm{~mm}$ of cancer), fusion biopsy failed to find the tumor. These patients remain at active monitoring.

\section{Discussion}

Prostate cancer (Gleason score $\geq 6$ ) was detected by fusion biopsy in $66 \%$ of the patients in our selected cohort. Almost all biopsies were considered representative showing that our novel local reference augmented method is feasible in clinical practice. Upgrading and new cancers diagnosed by repeat fusion biopsy after systematic biopsies had substantial impact on risk categories in our material. In $51 \%$ of all cases the management of patients was influenced. In 113 cases with no cancer after systematic biopsies, 71 had cancer diagnosed and half of those 113 were considered for curative treatment. In 91 patients with previously diagnosed low risk cancers $36 \%$ fulfilled the criteria for curative treatment.

MRI plays an increasingly important role in PCa diagnostics and is recommended in men with previously negative TRUS biopsy. In some national guidelines is now recommended prior to biopsy ${ }^{23}$. The optimal biopsy method after MRI is under discussion. Wegelin et al showed in a systematic review that none of 
these techniques were able to demonstrate significant differences between fusion biopsy and cognitive biopsy on overall cancer and clinically significant cancer detection ${ }^{24}$.

In our study we chose to use bpMRI instead of mpMRI because of several reasons.

MpMRI is both time consuming and it is more expensive than bpMRI and it also has been shown that gadolinium can form depositions in the brain ${ }^{25-27}$. In view of these findings, it seems to be advisable to reconsider the necessity of DCE.

It has been demonstrated that the sensitivity of bpMRI at 3T in the detection of PCa is similar to that of mpMRI and some investigators have found that DCE could potentially add to the false positive ${ }^{28,29}$. Kuhl et al. showed that bpMRI missed 1 out of 139 clinically significant cancer while mpMRI misclassified 11 cases as false positive ${ }^{28}$. Woo et al. reported a pooled sensitivity of 0.74 and a specificity of 0.90 for bpMRI and a pooled sensitivity of 0.76 and a specificity of 0.89 for mpMRI. Therefore, the authors conclude that bpMRI has the same diagnostic performance as mpMRI for the detection of $\mathrm{PCa}{ }^{30}$.

The cancer detection rate increased with increasing PI-RADS. Our radiologists identified 73 patients with PI-RADS 2-3. Among these we found 37 patients with positive biopsies of which 15 patients were diagnosed with GS 6 (ISUP 1). It has been shown in other studies that PI-RADS 2 lesions most likely do not represent clinically significant PCa and therefore ${ }^{31}$, later during the study, we chose PI-RADS 3 as cutoff. The fusion technique was shown to reduce the number of biopsies done on patients that have lowgrade $\mathrm{PCa}$, reducing the overall diagnoses of low-grade $\mathrm{PCa}$, and increasing the detection of the intermediate and high-risk subgroups of patients compared to traditional modalities ${ }^{32}$. Our study shows the same results.

Targeted biopsy yielded a significantly higher positivity rate for biopsy cores, and thus required significantly fewer cores to detect PCa and more importantly, significant PCa $9,32,33$. Our observation is in line with previous observations that repeat fusion guided biopsy gives a better higher yield of positive biopsies and better tumour infiltration of each core compared to repeat systematic biopsies ${ }^{32,34}$. The lesser number of cores needed for fusion guided biopsy decreases the risk of biopsy related complications.

The location of the tumour within the prostate has a significant impact on the diagnostic yield of standard 10-12 core biopsies. These sample predominantly the dorsal part of the gland and thus there is a risk of missing significant lesions in the anterior parts ${ }^{10,12}$. MRI can identify those lesions and fusionguided targeted biopsy will diagnose a significant number of them as shown in our study, further supporting the efficacy of the method. Most of the tumours were located anteriorly (54\%). Of these 228 patients with anteriorly tumours, standard biopsy revealed only $45(20 \%)$ patients of whom $82 \%$ were after MRI/TRUS guided biopsy upgraded to more significant cancer who also later went for curative therapy. These findings further corroborate the efficacy of fusion guided biopsies. This demands further systematic study beyond the scope of this paper. 
Urologists diagnosing PCa are aware of tumour heterogeneity and the importance of adequate sampling to appropriately assess the aggressiveness of the tumour. By utilizing a targeted approach to PCa detection, more tissue can be sampled from the lesions of interest as opposed to random biopsies, which would sample benign or clinically insignificant lesions. Several studies have shown the benefit of utilizing MRI/TRUS fusion guided prostate biopsy in the diagnosis of PCa 7,35 . Siddiqui and colleagues evaluated 1003 men undergoing both MRI/TRUS targeted biopsy and standard biopsy. They showed that MR/TRUS fusion biopsy was associated with increased detection of high-risk PCa and decreased detection of lowrisk PCa. The same study demonstrated MR/TRUS fusion biopsy to better predict final pathology on subsequent radical prostatectomy ${ }^{36}$.

Some weaknesses of our study should be mentioned. Due to inexperience for both radiologist and urologist the respective learning curves should be considered. Twenty-five early cases of the 423 were not adequately PI-RADS-classified which also could be due to radiologist learning curve. Since the classification was used anyway for biopsies, taken on an intention-to-to treat/biopsy basis, we decided against having a central review of all cases. The retrospective design should be acknowledged. Since our department receive referred patients from other hospitals the quality of MRI might vary. For the same reason the information regarding the number of previous biopsies was insufficient in some cases. There is no control group of patients who received a full multiparametric MRI protocol.

The much higher rate of significant PCa in MRI/TRUS fusion guided biopsies as well as the findings in the recent randomized trial ${ }^{9}$ poses on argument for primary fusion guided biopsy, omitting systematic biopsies.

\section{Conclusion}

MRI/TRUS fusion guided repeat biopsy, both for primary diagnosis and follow-up biopsies significantly increases diagnostic yields after systematic 10-12 core biopsy particularly in anterior tumours. A significant upgrading or newly diagnosed cases between standard 10-12 core and MRI/TRUS fusion guided biopsies provides better basis for therapeutic decisions.

\section{Declarations}

\section{Authors Contribution}

Michael Häggman (MH): Manuscript writing, Project development

Mats Ahlberg (MA): Data collection

Pär Dahlman (PD): Data collection, Image analysis

Per Liss (PL): Data collection, Image analysis

Anca Dragomir (AD): Pathology analysis 
Pontus Röbeck (PR): Data collection

Rafaele Cantera Ahlman (RA): Data collection

Håkan Jorulf (HJ): Project development

Sam Ladjevardi (SL): Manuscript writing, Data collection, Project development

\section{Acknowledgements}

Johan Westerbergh, for statistical analysis. Anna Tolf, Department of Pathology, Uppsala University for reviewing pathology specimens.

Funding: This research was funded by grants from Percy Falk.

Disclaimer. The funder had no role in the design and conduct of the study; collection, management, analysis, and interpretation of data; writing of the manuscript; or the decision to submit the manuscript for publication.

Disclosures: None of the authors claim to have any conflicts of interest as listed by the journal.

\section{Ethical considerations}

The planned study was subjected to review and approved by the Regional Ethical Board (Uppsala Dnr 2012-165 with amendment 2016 and 2019-00534) and conducted in accordance.

\section{Informed consent,}

It was judged by The Regional Ethical Board that no informed consent was needed for this retrospective study.

\section{References}

1. Ferlay J, Soerjomataram I, Dikshit R, et al. Cancer incidence and mortality worldwide: sources, methods and major patterns in GLOBOCAN 2012. Int J Cancer. 2015;136(5):E359-86.

2. a Mpalang RK, Boreux R, Melin P, Akir Ni Bitiang K, Daube G, De Mol P. Prevalence of Campylobacter among goats and retail goat meat in Congo. J Infect Dev Ctries. 2014;8(2):168-75.

3. McNeal JE, Redwine EA, Freiha FS, Stamey TA. Zonal distribution of prostatic adenocarcinoma. Correlation with histologic pattern and direction of spread. Am J Surg Pathol. 1988;12(12):897-906.

4. Reissigl A, Pointner J, Strasser H, Ennemoser O, Klocker H, Bartsch G. Frequency and clinical significance of transition zone cancer in prostate cancer screening. Prostate. 1997;30(2):130-5.

5. Kabalin JN, McNeal JE, Price HM, Freiha FS, Stamey TA. Unsuspected adenocarcinoma of the prostate in patients undergoing cystoprostatectomy for other causes: incidence, histology and 
morphometric observations. J Urol. 1989;141(5):1091-4. discussion 1093-1094.

6. Lundstrom KJ, Drevin L, Carlsson S, et al. Nationwide population based study of infections after transrectal ultrasound guided prostate biopsy. J Urol. 2014;192(4):1116-22.

7. Valerio M, Donaldson I, Emberton M, et al. Detection of Clinically Significant Prostate Cancer Using Magnetic Resonance Imaging-Ultrasound Fusion Targeted Biopsy: A Systematic Review. Eur Urol. 2015;68(1):8-19.

8. Nam RK, Saskin R, Lee Y, et al. Increasing hospital admission rates for urological complications after transrectal ultrasound guided prostate biopsy. J Urol. 2013;189(1 Suppl):12-7. discussion S17-18.

9. Rastinehad AR, Turkbey B, Salami SS, et al. Improving detection of clinically significant prostate cancer: magnetic resonance imaging/transrectal ultrasound fusion guided prostate biopsy. J Urol. 2014;191(6):1749-54.

10. Bott SR, Young MP, Kellett MJ, Parkinson MC. Contributors to the UCLHTRPD. Anterior prostate cancer: is it more difficult to diagnose? BJU Int. 2002;89(9):886-9.

11. Rastinehad AR, Abboud SF, George AK, et al. Reproducibility of Multiparametric Magnetic Resonance Imaging and Fusion Guided Prostate Biopsy: Multi-Institutional External Validation by a Propensity Score Matched Cohort. J Urol. 2016;195(6):1737-43.

12. Baco E, Rud E, Ukimura O, et al. Effect of targeted biopsy guided by elastic image fusion of MRI with 3D-TRUS on diagnosis of anterior prostate cancer. Urol Oncol. 2014;32(8):1300-7.

13. Rouviere $O$, Puech $P$, Renard-Penna R, et al. Use of prostate systematic and targeted biopsy on the basis of multiparametric MRI in biopsy-naive patients (MRI-FIRST): a prospective, multicentre, paired diagnostic study. Lancet Oncol. 2019;20(1):100-9.

14. Kasivisvanathan V, Rannikko AS, Borghi M, et al. MRI-Targeted or Standard Biopsy for ProstateCancer Diagnosis. N Engl J Med. 2018;378(19):1767-77.

15. Barentsz JO, Richenberg J, Clements R, et al. ESUR prostate MR guidelines 2012. Eur Radiol. 2012;22(4):746-57.

16. Vargas HA, Hotker AM, Goldman DA, et al. Updated prostate imaging reporting and data system (PIRADS v2) recommendations for the detection of clinically significant prostate cancer using multiparametric MRI: critical evaluation using whole-mount pathology as standard of reference. Eur Radiol. 2016;26(6):1606-12.

17. Di Campli E, Delli Pizzi A, Seccia B, et al. Diagnostic accuracy of biparametric vs multiparametric MRI in clinically significant prostate cancer: Comparison between readers with different experience. Eur $\mathrm{J}$ Radiol. 2018;101:17-23.

18. Kaplan I, Oldenburg NE, Meskell P, Blake M, Church P, Holupka EJ. Real time MRI-ultrasound image guided stereotactic prostate biopsy. Magn Reson Imaging. 2002;20(3):295-9.

19. Rud E, Baco E, Eggesbo HB. MRI and ultrasound-guided prostate biopsy using soft image fusion. Anticancer Res. 2012;32(8):3383-9. 
20. Natarajan S, Marks LS, Margolis DJ, et al. Clinical application of a 3D ultrasound-guided prostate biopsy system. Urol Oncol. 2011;29(3):334-42.

21. Verma S, Choyke PL, Eberhardt SC, et al. The Current State of MR Imaging-targeted Biopsy Techniques for Detection of Prostate Cancer. Radiology. 2017;285(2):343-56.

22. Bjurlin MA, Mendhiratta N, Wysock JS, Taneja SS. Multiparametric MRI and targeted prostate biopsy: Improvements in cancer detection, localization, and risk assessment. Cent European J Urol. 2016;69(1):9-18.

23. Cancer SNgfP. https://www.cancercentrum.se/samverkan/cancerdiagnoser/prostata/vardprogram/. 2020.

24. Wegelin O, van Melick HHE, Hooft L, et al. Comparing Three Different Techniques for Magnetic Resonance Imaging-targeted Prostate Biopsies: A Systematic Review of In-bore versus Magnetic Resonance Imaging-transrectal Ultrasound fusion versus Cognitive Registration. Is There a Preferred Technique? Eur Urol. 2017;71(4):517-31.

25. Obmann VC, Pahwa S, Tabayayong W, et al. Diagnostic Accuracy of a Rapid Biparametric MRI Protocol for Detection of Histologically Proven Prostate Cancer. Urology. 2018;122:133-8.

26. Junker D, SteinkohI F, Fritz V, et al. Comparison of multiparametric and biparametric MRI of the prostate: are gadolinium-based contrast agents needed for routine examinations? World J Urol. 2019;37(4):691-9.

27. McDonald RJ, McDonald JS, Kallmes DF, et al. Intracranial Gadolinium Deposition after Contrastenhanced MR Imaging. Radiology. 2015;275(3):772-82.

28. Kuhl CK, Bruhn R, Kramer N, Nebelung S, Heidenreich A, Schrading S. Abbreviated Biparametric Prostate MR Imaging in Men with Elevated Prostate-specific Antigen. Radiology. 2017;285(2):493505.

29. Stanzione A, Imbriaco M, Cocozza S, et al. Biparametric 3T Magnetic Resonance Imaging for prostatic cancer detection in a biopsy-naive patient population: a further improvement of PI-RADS v2? Eur J Radiol. 2016;85(12):2269-74.

30. Woo S, Suh CH, Kim SY, Cho JY, Kim SH, Moon MH. Head-to-Head Comparison Between Biparametric and Multiparametric MRI for the Diagnosis of Prostate Cancer: A Systematic Review and MetaAnalysis. AJR Am J Roentgenol. 2018;211(5):W226-41.

31. Liddell H, Jyoti R, Haxhimolla HZ. mp-MRI Prostate Characterised PIRADS 3 Lesions are Associated with a Low Risk of Clinically Significant Prostate Cancer - A Retrospective Review of 92 Biopsied PIRADS 3 Lesions. Curr Urol. 2015;8(2):96-100.

32. Baco E, Rud E, Eri LM, et al. A Randomized Controlled Trial To Assess and Compare the Outcomes of Two-core Prostate Biopsy Guided by Fused Magnetic Resonance and Transrectal Ultrasound Images and Traditional 12-core Systematic Biopsy. Eur Urol. 2016;69(1):149-56.

33. Maxeiner A, Fischer T, Stephan C, et al. [Real-time MRI/US fusion-guided biopsy improves detection rates of prostate cancer in pre-biopsied patients]. Aktuelle Urol. 2014;45(3):197-203. 
34. Boesen L, Norgaard N, Logager V, Balslev I, Thomsen HS. Multiparametric MRI in men with clinical suspicion of prostate cancer undergoing repeat biopsy: a prospective comparison with clinical findings and histopathology. Acta Radiol. 2018;59(3):371-80.

35. Boesen L, Norgaard N, Logager V, Balslev I, Thomsen HS. Where Do Transrectal Ultrasound- and Magnetic Resonance Imaging-guided Biopsies Miss Significant Prostate Cancer? Urology. 2017;110:154-60.

36. Siddiqui MM, Rais-Bahrami S, Turkbey B, et al. Comparison of MR/ultrasound fusion-guided biopsy with ultrasound-guided biopsy for the diagnosis of prostate cancer. JAMA. 2015;313(4):390-7.

\section{Tables}

Please see the supplementary files section to view the tables.

\section{Supplementary Files}

This is a list of supplementary files associated with this preprint. Click to download.

- tables.docx

- tables.docx

- tables.docx 